\title{
Xenon-129 MRI detects ventilation deficits in paediatric stem cell transplant patients unable to perform spirometry
}

\author{
Laura L. Walkup (10 ${ }^{1,2,3}$, Kasiani Myers ${ }^{2,4}$, Javier El-Bietar ${ }^{2,4,+}{ }^{\text {Adam Nelson }}{ }^{2,4}$, \\ Matthew M. Willmering (1) ${ }^{1}$, Michael Grimley ${ }^{2,4}$, Stella M. Davies ${ }^{2,4}$, \\ Christopher Towe ${ }^{2,3}$ and Jason C. Woods ${ }^{1,2,3}$
}

Affiliations: ${ }^{1}$ Center for Pulmonary Imaging Research, Division of Pulmonary Medicine and Dept of Radiology, Cincinnati Children's Hospital Medical Center, Cincinnati, OH, USA. ${ }^{2}$ Dept of Pediatrics, College of Medicine, University of Cincinnati, Cincinnati, OH, USA. ${ }^{3}$ Division of Pulmonary Medicine, Cincinnati Children's Hospital Medical Center, Cincinnati, OH, USA. "Division of Bone Marrow Transplantation and Immune Deficiency, Cincinnati Children's Hospital Medical Center, Cincinnati, OH, USA.

Correspondence: Jason C. Woods, Cincinnati Children's Hospital Medical Center, 3333 Burnet Avenue, MLC 5033, Cincinnati, OH 45229, USA. E-mail: Jason.woodsacchmc.org

@ERSpublications

Hyperpolarised ${ }^{129} \mathrm{Xe}$ MRI detects regional lung ventilation abnormalities in paediatric stem cell transplantation patients, including in patients with normal spirometry and in patients unable to perform reliable spirometry http://ow.ly/1T2030nGGQ1

Cite this article as: Walkup LL, Myers K, El-Bietar J, et al. Xenon-129 MRI detects ventilation deficits in paediatric stem cell transplant patients unable to perform spirometry. Eur Respir J 2019; 53: 1801779 [https://doi.org/10.1183/13993003.01779-2018].

\section{ABSTRACT}

Background: Early detection of pulmonary morbidity following haematopoietic stem cell transplantation (HSCT) remains an important challenge for intervention, primarily due to the insensitivity of spirometry to early change, and in paediatrics, patient compliance provides additional challenges. Regional lung ventilation abnormalities in paediatric HSCT patients were quantified using hyperpolarised xenon-129 $\left({ }^{129} \mathrm{Xe}\right)$ magnetic resonance imaging (MRI) and compared to spirometry.

Methods: Medically stable, paediatric allogeneic HSCT patients $(n=23$, ages $6-16$ years) underwent an outpatient MRI scan where regional ventilation was quantified with a breath-hold of hyperpolarised ${ }^{129} \mathrm{Xe}$ gas. Ventilation deficits, regions of the lung that ventilate poorly due to obstruction, were quantified as a ventilation defect percentage (VDP) and compared to forced expiratory volume in $1 \mathrm{~s}$ (FEV1), FEV1/forced vital capacity (FVC) ratio, and forced expiratory flow at $25-75 \%$ of $\mathrm{FVC}$ (FEF25-75\%) from spirometry using linear regression.

Results: The mean \pm SD ${ }^{129} \mathrm{Xe}$ VDP was $10.5 \pm 9.4 \%$ (range $2.6-41.4 \%$ ). ${ }^{129} \mathrm{Xe}$ VDP correlated with FEV1, $\mathrm{FEV} 1 / \mathrm{FVC}$ ratio and $\mathrm{FEF}_{25}-75 \%$ ( $\mathrm{p} \leqslant 0.02$ for all comparisons). Ventilation deficits were detected in patients with normal spirometry (i.e. FEV1 $>80 \%$ ), supporting the sensitivity of ${ }^{129} \mathrm{Xe}$ MRI to early obstruction reported in other pulmonary conditions. Seven (30\%) patients could not perform spirometry, yet ventilation deficits were observed in five of these patients, detecting abnormalities that otherwise may have gone undetected and untreated until advanced.

Conclusion: Lung ventilation deficits were detected using hyperpolarised ${ }^{129}$ Xe gas MRI in asymptomatic paediatric HSCT patients and in a subgroup who were unable to perform reliable spirometry. ${ }^{129} \mathrm{Xe}$ MRI provides a reliable imaging-based assessment of pulmonary involvement in this potentially difficult to diagnose paediatric population. 


\section{Introduction}

Pulmonary complications following allogeneic haematopoietic stem-cell transplantation (HSCT) are a significant source of morbidity and mortality, affecting up to $60 \%$ of all HSCT patients, with the highest prevalence in patients with graft versus host disease (GVHD) [1, 2]. These complications may arise early or later post-transplantation (i.e. $>100$ days) and may stem from bacterial or fungal infections or from non-infectious sources such as pulmonary oedema or drug-related toxicity related to immunosuppression and chemotherapy [2,3]. Bronchiolitis obliterans syndrome (BOS), the most common and severe later-onset pulmonary complication following HSCT, is an obstructive lung condition resulting from a combination of immune-mediated inflammation and fibrosis in the small airways. BOS is associated with precipitous declines in pulmonary function, high respiratory morbidity and eventual mortality. Commonly, BOS is an irreversible process, and while there are limited therapies available to stabilise lung function, the only treatment for end-stage lung disease is lung transplantation, which is also associated with BOS and poor prognosis.

Routine pulmonary function testing (PFT) such as spirometry is traditionally the first line of detection of lung abnormalities. The primary clinical parameter from spirometry is the forced expiratory volume in $1 \mathrm{~s}$ (FEV1), but generally the diagnosis of BOS is made only after significant consistent decline in FEV1. The clinical symptoms of BOS include chronic nonproductive cough, wheezing, exercise intolerance and dyspnoea on exertion; patients with these symptoms often already have moderate to severe FEV1 declines [4]. Trajectories of FEV1 following HSCT have shown the steepest decline in the 6 months prior to BOS diagnosis followed by stabilisation after diagnosis and intervention [5]. A multicentre, retrospective study of adult HSCT patients in the UK showed that $10 \%$ had significant decline in FEV1 and forced vital capacity (FVC) at 12 months post-HSCT compared to pre-transplantation [6], and in another study, UHLVING et al. [7] reported that $62 \%$ of their paediatric HSCT cohort had reductions in lung function of $>10 \%$ in the first 3-9 months post-transplantation. Early detection of lung abnormalities is paramount for intervention to conserve pulmonary capacity and improve outcomes [5, 8, 9]. While spirometry is clinically ubiquitous and easy to deploy, there are known limitations, including a poor sensitivity to early disease [10, 11]. Even though routine spirometric screening of HSCT patients is recommended [12, 13], PrAIs et al. [14] suggest that protocols and fulfillment vary greatly across centres as evidenced by many retrospective studies of PFTs in HSCT patients with varying amounts of data and patients at each time point. Furthermore, in the paediatric population, compliance with the effort-dependent spirometry may be challenging. LoEB et al. [15] assessed the acceptability and repeatability of spirometry in paediatric subjects and reported that only $50 \%$ of subjects at the age of 6 years could perform acceptable repeatable spirometry, which rose to $85 \%$ by 10 years.

In addition to spirometry, clinical imaging such as chest radiography computed tomography (CT) may be pursued to detect lung disease. The characteristics of BOS on high-resolution chest CT include mosaic patterning, bronchiectasis, atelectasis and in later stages, severe air trapping [16]; however, these features are typically subtle in early disease. Concerns for exposure to ionising radiation in the paediatric population require judicious use of chest CT, limiting its application for routine screening of asymptomatic patients. While there are efforts to minimise ionising radiation exposure, including modern low-dose CT protocols and expiration-only CT protocols [17], a more sensitive, non-ionising imaging modality would allow for more frequent assessment of lung disease progression or therapeutic response.

Over the past 20 years, the sensitivity and specificity of hyperpolarised-gas magnetic resonance imaging (MRI) to detect early lung obstruction in asymptomatic patients has been demonstrated across a wide variety of pulmonary conditions including cystic fibrosis [18, 19], asthma [20-22], interstitial lung disease [23] and chronic obstructive pulmonary disease $[21,24,25]$. In hyperpolarised xenon-129 $\left({ }^{129} \mathrm{Xe}\right) \mathrm{MRI}$ techniques, the signal of ${ }^{129} \mathrm{Xe}$ gas is enhanced many orders of magnitude over thermal equilibrium via spin exchange optical pumping, such that the gas may be inhaled and imaged via MRI during a single breath-hold (i.e. generally $\geqslant 5 \mathrm{~s}$ ). Regions of the lung that ventilate appear bright in the ${ }^{129} \mathrm{Xe}$ images, and regions that are partially or fully obstructed appear relatively or completely dark, respectively, due to the inability of ${ }^{129} \mathrm{Xe}$ gas to fill those obstructed airspaces. These deficits in lung ventilation can be quantified, and the repeatability and stability of hyperpolarised-gas ventilation deficits have been demonstrated [26-28], supporting outcomes from ${ }^{129} \mathrm{Xe}$ MRI as biomarkers for obstructive lung disease. Indeed, hyperpolarised-gas MRI has been reported in lung transplantation; in a preliminary report of helium-3 $\left({ }^{3} \mathrm{He}\right)$ MRI in six adult lung-transplantation recipients, McAdAms et al. [29] found that the extent of ${ }^{3} \mathrm{He}$ ventilation defects correlated with the severity of BOS. GAST et al. [30] used ${ }^{3} \mathrm{He}$ ventilation and oxygen-sensitive MRI methods to investigate normal lung grafts and those with BOS and found that normal grafts had fewer ventilation defects and BOS patients had more heterogeneous intrapulmonary oxygen distribution.

We hypothesised that ${ }^{129} \mathrm{Xe}$ MRI could detect ventilation abnormalities in a paediatric HSCT population, including in children who could not perform reliable spirometry, and that quantitative ${ }^{129} \mathrm{Xe}$ ventilation 
would be sensitive to lung abnormalities post-HSCT before changes via traditional spirometry (i.e. ${ }^{129} \mathrm{Xe}$ ventilation deficits would be present in HSCT patients with normal spirometry), providing a means of early detection and intervention. Portions of this work have been presented previously in abstract form [31, 32].

\section{Methods}

\section{Subjects and ${ }^{129} \mathrm{Xe}$ gas preparation}

23 paediatric allogeneic HSCT recipients were recruited for the ${ }^{129}$ Xe MRI study with institutional review board approval following United States Food and Drug Administration (US FDA) investigational new drug (IND) approval (IND number 123577). Inclusion criteria included age $\geqslant 6$ years (lower limit of the IND) and ability to perform a breath-hold. Table 1 summarises the demographics for this cohort. In addition to standard MRI exclusion criteria (e.g. claustrophobia, incompatible implants), additional exclusions included symptoms of current respiratory infection (loose or productive cough or wheeze), chest tightness within the previous week, baseline pulse oximetry $<95 \%$ and/or positive pregnancy test (if applicable). All subjects were medically stable outpatients at the time of imaging. Isotopically enriched Xe gas $\left(86 \%{ }^{129} \mathrm{Xe}\right)$ was polarised to $\sim 20 \%$ using a commercial polariser (Polarean Imaging, Durham, NC, USA) and dispensed into a Tedlar delivery bag (Jensen Inert Products, Coral Springs, FL, USA) equipped with Tygon tubing, plastic hose clamp and a mouthpiece (Epsilon Medical Devices, Penang, Malaysia).

\section{MRI procedure}

After screening, subjects were placed supine in a Philips (Best, the Netherlands) 3 Tesla Achieva MRI scanner with a homebuilt ${ }^{129} \mathrm{Xe}$ saddle coil tuned to $35.3 \mathrm{MHz}$. Standard three-plane, hydrogen-1 $\left({ }^{1} \mathrm{H}\right)$ localisation scans were performed first to ensure optimal field of view for lung imaging. Next, a conventional ${ }^{1} \mathrm{H}$ gradient-echo scan was performed with a practice breath-hold of room air. For all breath-holds (i.e. ${ }^{1} \mathrm{H}$ and ${ }^{129} \mathrm{Xe}$ ), the gas administrator instructed the subject to fully inhale and exhale twice before gas inhalation beginning at functional residual capacity. The maximum scan duration was $16 \mathrm{~s}$ for all scans requiring a breath-hold, and the subject was in the MRI scanner for $\sim 15 \mathrm{~min}$ for hyperpolarised ${ }^{129} \mathrm{Xe}$ MRI. Due to the non-renewable, hyperpolarised nature of the ${ }^{129} \mathrm{Xe}$ gas, practice breath-holds with room air were repeated if necessary to ensure compliance for the ${ }^{129} \mathrm{Xe}$ scans.

First, a small calibration dose of $\sim 250 \mathrm{~mL}$ of hyperpolarised ${ }^{129} \mathrm{Xe}$ gas was administered during a brief 2-s breath-hold to optimise the in vivo flip angle for the ${ }^{129} \mathrm{Xe}$ ventilation images. For ${ }^{129} \mathrm{Xe}$ ventilation imaging, the ${ }^{129} \mathrm{Xe}$ gas dose was one-sixth of a subject's predicted total lung capacity as calculated from plethysmography-based predictive equations using a subject's sex and height [33]. ${ }^{129}$ Xe ventilation was acquired using a gradient-echo scan $\left(9-12^{\circ}\right.$ flip angle, repetition time $=8 \mathrm{~ms}$, echo time $=4 \mathrm{~ms}$, nine to 15 slices depending on subject's size, and voxel size $\left.\approx 3 \times 3 \times 15 \mathrm{~mm}^{3}[34]\right)$.

${ }^{129} \mathrm{Xe}$ gas was administered in the presence of a medical professional (i.e. registered nurse or physician), and a minimum of $2 \mathrm{~min}$ elapsed between consecutive ${ }^{129} \mathrm{Xe}$ breath-holds. Subject blood oxygenation (arterial oxygen saturation measured by pulse oximetry $\left(\mathrm{SpO}_{2}\right)$ ) and heart-rate were monitored throughout the ${ }^{129} \mathrm{Xe}$ breath-holds using a magnetic resonance-compatible pulse oximeter (InVivo Corporation, Orlando, FL, USA), and changes in vitals were compared to baseline resting values using paired t-tests with a p-value $\leqslant 0.05$ considered significant. Adverse events were assessed during the ${ }^{129} \mathrm{Xe}$ MRI procedure and via follow-up phone call at day 1 and day 30 ( \pm 7 days).

\footnotetext{
${ }^{129}$ Xe ventilation analysis

${ }^{129} \mathrm{Xe}$ ventilation images were analysed using custom software in MATLAB (MathWorks, Natick, MA, USA). Lung masks were generated using ${ }^{1} \mathrm{H}$ MRI to define the edges of the lungs excluding large airways and vasculature. The ${ }^{129} \mathrm{Xe}$ ventilation defect percentage (VDP) was calculated using a threshold of $<60 \%$ of the mean whole-lung ${ }^{129} \mathrm{Xe}$ signal and quantified as a percentage of the total lung volume. VDP calculated in this manner is a well-established, reproducible outcome measure in the hyperpolarised-gas MRI literature [35-39]. This $60 \%$ threshold has been used previously to best separate healthy paediatric controls from those with lung obstruction in cystic fibrosis [40]. ${ }^{129} \mathrm{Xe}$ VDP was compared to a cohort of age-matched control subjects ( $\mathrm{n}=10$; seven males and three females) with a mean \pm sD age of $12 \pm 3$ years (range 6-16 years) and $\mathrm{FEV}_{1} \%$ predicted of $102 \pm 9 \%$ (range $89-115 \%$ ), which were published previously [40]. Ventilation deficits were considered present if ${ }^{129} \mathrm{Xe}$ VDP was $>6 \%$, which is the upper limit typical of our control subjects. ${ }^{129} \mathrm{Xe}$ image results were compared to measures from FEV1, FEV1/FVC ratio and forced expiratory flow at $25-75 \%$ of $\mathrm{FVC}\left(\mathrm{FEF}_{25}-75 \%\right)$ from spirometry when available using linear regression and Pearson's correlations. Clinical spirometry reports were collected from patient medical records if within 6 months of the MRI. If no recent clinical spirometry was available, spirometry was attempted on the day of the MRI visit.
} 


\section{TABLE 1 Patient demographics}

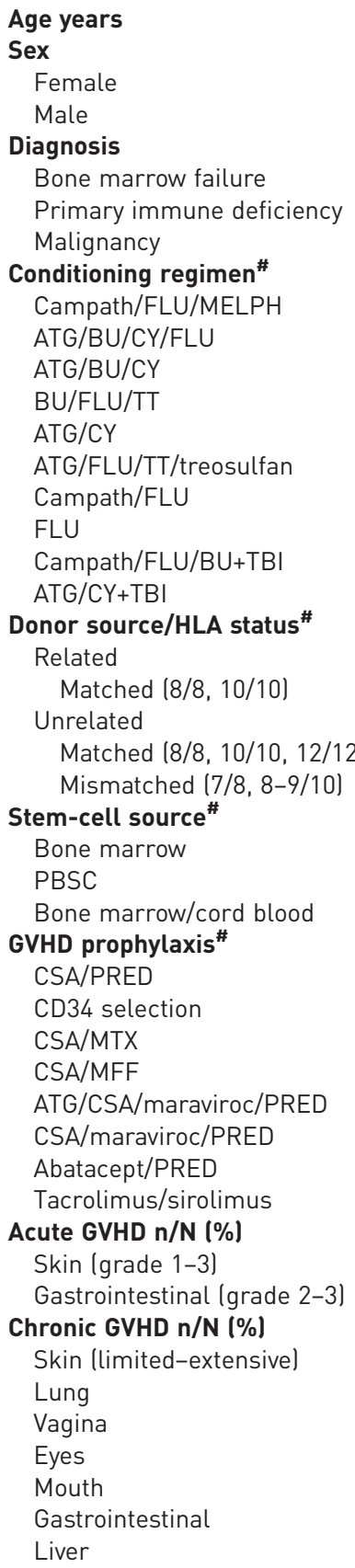

Data are presented as mean (range), $\mathrm{n}(\%)$ or $\mathrm{n}$, unless otherwise stated. FLU: fludarabine; MELPH melphalan; ATG: antithymocyte globulin; BU: busulfan; CY: cyclophosphamide; TT: thiotepa; TBI: total body irradiation; HLA: human leukocyte antigen; PBSC: peripheral blood stem cell; GVHD: graft versus host disease; CSA: cyclosporine; PRED: prednisone; MTX: methotrexate; MFF: mycophenolate mofetil. \#: $n=24$ transplantations, as one subject underwent two haematopoietic stem cell transplantations.

\section{Results}

The ${ }^{129} \mathrm{Xe}$ MRI procedure was well tolerated and completed by all subjects. Table 2 summarises the changes in $\mathrm{SpO}_{2}$ and heart rate during the calibration and ventilation imaging doses of Xe gas. As anticipated, there was a small, short decrease in $\mathrm{SpO}_{2}$ associated with the ventilation dose of gas, a mean decrease of $\sim 8 \%$ from baseline (compared to $\sim 6 \%$ decrease reported for paediatric cystic fibrosis patients and healthy controls [34]). Of the 19 subjects who had a decrease in $S_{\mathrm{pO}_{2}}$, the duration of this nadir was $<10 \mathrm{~s}$ and $\mathrm{SpO}_{2}$ 
TABLE 2 Blood oxygenation and heart rate changes during xenon-129 magnetic resonance imaging

\begin{tabular}{|c|c|c|c|c|c|c|}
\hline & \multicolumn{3}{|c|}{ Flip-angle calibration dose } & \multicolumn{3}{|c|}{ Ventilation-imaging dose } \\
\hline $\mathrm{SpO}_{2} \%$ & $\begin{array}{l}97.4 \pm 1.5 \\
(95-100)\end{array}$ & $\begin{array}{l}93.5 \pm 4.2 \\
(86-104)\end{array}$ & $\begin{array}{l}97.5 \pm 1.7 \\
(93-100)\end{array}$ & $\begin{array}{l}97.2 \pm 1.4 \\
(94-100)\end{array}$ & $\begin{array}{c}89.3 \pm 5.2 \\
(74-97)\end{array}$ & $\begin{array}{l}98.0 \pm 1.6 \\
(94-100)\end{array}$ \\
\hline$p$-value & & 0.0018 & 0.74 & & $1 \times 10^{-6}$ & 0.009 \\
\hline Heart rate beats $\cdot \min ^{-1}$ & $\begin{array}{c}92 \pm 14 \\
(74-109)\end{array}$ & $\begin{array}{c}93 \pm 11 \\
(82-121)\end{array}$ & $\begin{array}{c}92 \pm 13 \\
(70-116)\end{array}$ & $\begin{array}{c}92 \pm 13 \\
(68-124)\end{array}$ & $\begin{array}{c}96 \pm 13 \\
(66-123)\end{array}$ & $\begin{array}{c}90 \pm 15 \\
(67-125)\end{array}$ \\
\hline
\end{tabular}

Data are presented as mean \pm SD (range), unless otherwise stated. $\mathrm{SpO}_{2}$ : arterial oxygen saturation measured by pulse oximetry.

was restored for all subjects with normal breathing of room air. There were no related adverse events and no adverse events during the study visit that required medical intervention, in agreement with previously published safety assessments of ${ }^{129} \mathrm{Xe}$ MRI in paediatric [34] and adult subjects $[25,41]$.

The ${ }^{129}$ Xe ventilation pattern varied widely across subjects, as demonstrated in figure 1, including across patients with similar $\mathrm{FEV}_{1} \%$ pred values (figure $1 \mathrm{~b}, \mathrm{c}$ ). Ventilation deficits were apparent (i.e. VDP $>6 \%$ ) in $11(48 \%)$ subjects. The mean \pm SD ${ }^{129} \mathrm{Xe}$ VDP was $10.5 \pm 9.4 \%$ in all HSCT patients (range 2.6-41.4\%), which was elevated relative to controls [40] $(6.3 \pm 2.8 \%)$, but did not reach statistical significance $(p=0.06)$. A ${ }^{129} \mathrm{Xe}$ VDP threshold between $60 \%$ and $70 \%$ provided the maximum separation between HSCT subjects with BOS and control subjects. The wide individual variation in ventilation was apparent especially when ${ }^{129} \mathrm{Xe}$ VDP was plotted against FEV1 \% pred, FEV1/FVC ratio and FEF25-75\% (figure 2), as subjects with similar spirometry values had large differences in VDP. Reliable spirometry data were available for 16 out of the 23 subjects, and the mean \pm SD days between spirometry and ${ }^{129} \mathrm{Xe}$ MRI was $46 \pm 43$ days (range $0-137$ days). ${ }^{129}$ Xe VDP correlated with FEV1 \% pred with a p-value of 0.02; Pearson's coefficient -0.56 , with FEV1/FVC ratio $\left(\mathrm{p}<10^{-6}\right.$, Pearson's coefficient -0.92$)$, and FEF25-75\% ( $\mathrm{p}=0.0005$ and Pearson's coefficient -0.78).

Importantly, seven (30\%) out of 23 subjects in this study were unable to perform reliable posttransplantation spirometry due to technique; however, all subjects were able to complete the ${ }^{129} \mathrm{Xe}$ MRI protocol. As anticipated, the subgroup without reliable spirometry included primarily the youngest subjects, with an average age of $8 \pm 3$ years (range $6-13$ years). In this group the ${ }^{129} \mathrm{Xe}$ VDP was $11.4 \pm 8.4 \%$ (range 3.3-28\%) and ${ }^{129} \mathrm{Xe}$ ventilation deficits were apparent (i.e. VDP $>6 \%$ ) in five out of seven subjects. Reliable pre-transplantation FEV1 \% pred was available for only eight subjects, and post-transplantation FEV 1 was available for six of these subjects with an average decrease of $8 \%$ (range $0-32 \%$ ) at the time of ${ }^{129}$ Xe MRI. Patients with reliable spirometry $(n=16)$ were significantly older (average $12 \pm 3$ years, range 6-17 years; $\mathrm{p}=0.008$ ) and the ${ }^{129} \mathrm{Xe}$ VDP was $10.2 \pm 10.3 \%$ (range $3-41.4 \%$; nonsignificant). There was no significant difference in ${ }^{129} \mathrm{Xe}$ VDP between patients with known chronic GVHD of any system ( $\mathrm{n}=8$, average ${ }^{129} \mathrm{Xe}$ VDP $\left.10.6 \pm 7.8 \%\right)$ and those without chronic GVHD (10.5 $\left.\pm 10.4 \%\right)$. In the four patients with clinically diagnosed lung GVHD, ${ }^{129} \mathrm{Xe}$ VDP ranged from $5.5 \%$ to $24 \%$ (average $13.7 \pm 9.1 \%$ ), which was not statistically different from patients with other forms of chronic GVHD or from patients without chronic GVHD.

To evaluate early and late ventilation abnormalities after HSCT, ${ }^{129} \mathrm{Xe}$ VDP was compared to the number of days post-HSCT (figure 3a). Ventilation deficits were identified early and late after HSCT, but in the subgroup of 13 patients who had MRI within the first year post-HSCT (figure 3b), seven (54\%) patients had obvious ventilation deficits and ${ }^{129} \mathrm{Xe}$ VDP $>6 \%$, including several of the patients who were unable to perform reliable spirometry and one patient with normal FEV1 (figure 1c).

\section{Discussion}

To our knowledge, this is the first demonstration of hyperpolarised ${ }^{129} \mathrm{Xe}$ MRI in the HSCT population. ${ }^{129} \mathrm{Xe}$ MRI detected a wide range of lung ventilation abnormalities in paediatric HSCT patients. Ventilation deficits via ${ }^{129} \mathrm{Xe}$ MRI were detected in asymptomatic HSCT patients with normal FEV1 (i.e. $>80 \%$ predicted, e.g. figure 1c), which is in agreement with previous studies of ${ }^{129} \mathrm{Xe} \mathrm{MRI}$ in other pulmonary diseases with mild obstruction $[19,36,38,40]$. These results are in agreement with a case study of lung scintigraphy of an adult HSCT patient with relatively unremarkable chest CT who was diagnosed with bronchiolitis obliterans by the presence of matched ventilation-perfusion deficits on scintigraphy, supporting the diagnostic value of functional lung imaging in the HSCT population [42]. Longitudinal 


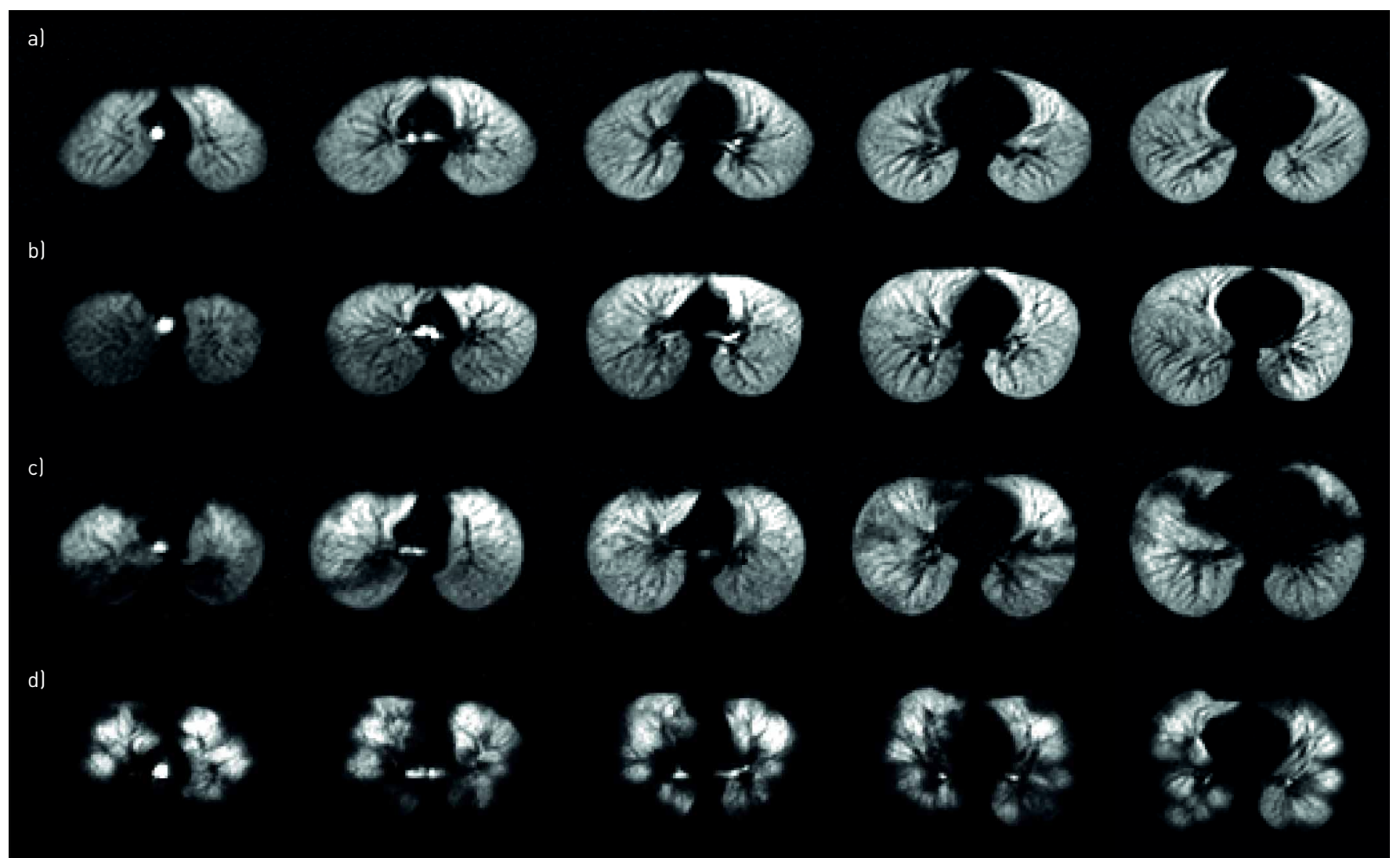

FIGURE 1 Representative axial xenon-129 ( $\left.{ }^{129} \mathrm{Xe}\right)$ ventilation magnetic resonance images from four haematopoietic stem cell transplantation (HSCT) patients demonstrating the range of ${ }^{129} \mathrm{Xe}$ ventilation patterns seen in this study. a) 15-year-old female, 145 days post-HSCT, forced expiratory volume in $1 \mathrm{~s}$ (FEV1) $108 \%$ predicted, ${ }^{129} \mathrm{Xe}$ ventilation defect percentage (VDP) $3.0 \%$; the subject has high $\mathrm{FEV} 1 \%$ pred, ventilation is homogenous and the ${ }^{129} \mathrm{Xe} \mathrm{VDP}$ is correspondingly low. The sensitivity of ${ }^{129} \mathrm{Xe}$ MRI to mild lung abnormalities is demonstrated in the subjects in b) (15-year-old female, 3 years post-HSCT, FEV $181 \%$ pred, ${ }^{129}$ Xe VDP $2.6 \%$ ) and c) (13-year-old female, 93 days post-HSCT, FEV $181 \%$ pred, ${ }^{129} \mathrm{Xe}$ VDP $20 \%$ ), both of whom have similar high FEV $1 \%$ pred, yet the subject in c) has large focal deficits (e.g. posterior left and right lungs near apexes, anterior right lung near the base) and higher VDP. d) 13 -year-old female, 9 years post-HSCT, FEV $152 \%$ pred, ${ }^{129}$ Xe VDP $41.4 \%$. ${ }^{129} \mathrm{Xe}$ ventilation is very heterogeneous with large deficits and correspondingly high ${ }^{129} \mathrm{Xe}$ VDP and low FEV $1 \%$ pred.

studies to understand how ${ }^{129} \mathrm{Xe}$ MRI may play a role in screening bone marrow transplant patients for future BOS risk are needed; however, this small, cross-sectional study demonstrates hyperpolarised ${ }^{129} \mathrm{Xe}$ MRI as a safe, feasible and sensitive modality in paediatric HSCT patients, even relatively soon (i.e. 100 days) post-transplantation, and even in children who are unable to perform reliable spirometry, a vastly underevaluated population.

While ${ }^{129} \mathrm{Xe}$ VDP correlated with FEV1, it is important to note that $\mathrm{FEV}_{1}$ is known to be somewhat insensitive to early obstruction [43,44]. Consistent with this were two HSCT subjects who had normal FEV 1 but $>6 \%$ VDP, suggesting that ${ }^{129} \mathrm{Xe}$ MRI is sensitive to early lung involvement in asymptomatic subjects. The correlation between ${ }^{129} \mathrm{Xe}$ VDP and FEV1/FVC ratio $\left(\mathrm{p}<10^{-6}\right)$ was stronger than the correlations between ${ }^{129} \mathrm{Xe}$ VDP and FEV1 $(p=0.02)$ and $\mathrm{FEF}_{25-75 \%}(\mathrm{p}=0.0005)$; this is in agreement with KIRBY et al. [38], who reported a stronger correlation between ${ }^{129} \mathrm{Xe}$ VDP and FEV1/FVC ratio than with FEV1. While FEV1 is the gold standard for assessing lung disease and reductions in FEV1 are associated with obstruction, it represents just one functional component of lung disease. FEV1/FVC ratio is a more specific marker for early airway involvement; this is consistent with the stronger correlation with VDP.

It has been reported that lower pre-transplantation $\mathrm{FEV}_{1}$ is associated with increased risk of pulmonary complications following HSCT [45]; however, this baseline is not always obtainable in paediatric subjects, which severely limits surveillance following transplantation. Without a reliable metric for surveillance, complications may go undetected and untreated, as was seen in this study, in the five subjects who could not perform spirometry, yet ${ }^{129} \mathrm{Xe}$ ventilation deficits were detected. Without pre-transplantation assessment via PFTs or imaging, it is unknown whether these ventilation abnormalities were pre-existing and reflective of underlying lung disease or occurred after HSCT, and this is one shortcoming of this study, in addition to the lack of same-day spirometry and ${ }^{129} \mathrm{Xe}$ MRI assessment. While spirometry is easily deployable, the paucity 

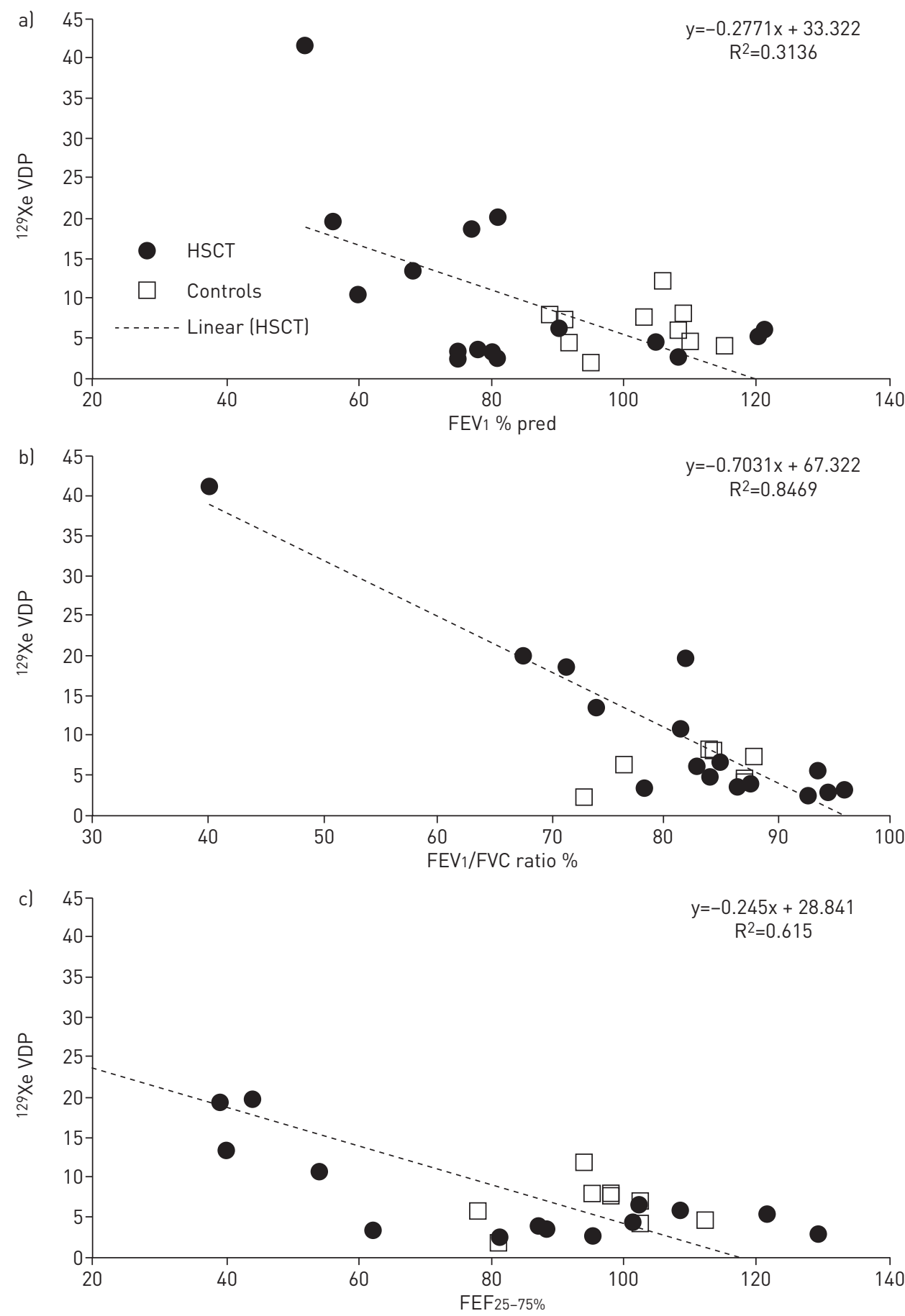

FIGURE 2 Xenon-129 ( $\left.{ }^{129} \mathrm{Xe}\right)$ ventilation defect percentage (VDP) versus a) forced expiratory volume in $1 \mathrm{~s}$ (FEV1) \% predicted; b) FEV1/forced vital capacity (FVC) ratio; c) forced expiratory flow at $25-75 \%$ of FVC (FEF25-75\%). HSCT: haematopoietic stem cell transplantation.

of reliable spirometry data in this study reinforces the notion that more robust and sensitive metrics for assessing paediatric lung disease are needed. Indeed, emerging PFTs such as lung clearance index via multiple-breath washout and impulse forced oscillometry have shown promise as being less effort-dependent and easier for paediatrics; however, these techniques have not yet been reported in the paediatric HSCT population. Hyperpolarised ${ }^{129}$ Xe MRI can address this need while providing additional spatial resolution which may be leveraged for targeted evaluations such as bronchoscopy and lung biopsy.

Currently hyperpolarised ${ }^{129} \mathrm{Xe}$ gas is regulated by the US FDA as an investigational drug, so its use is limited to research centres with expertise and specialised equipment. However, as the high translational 

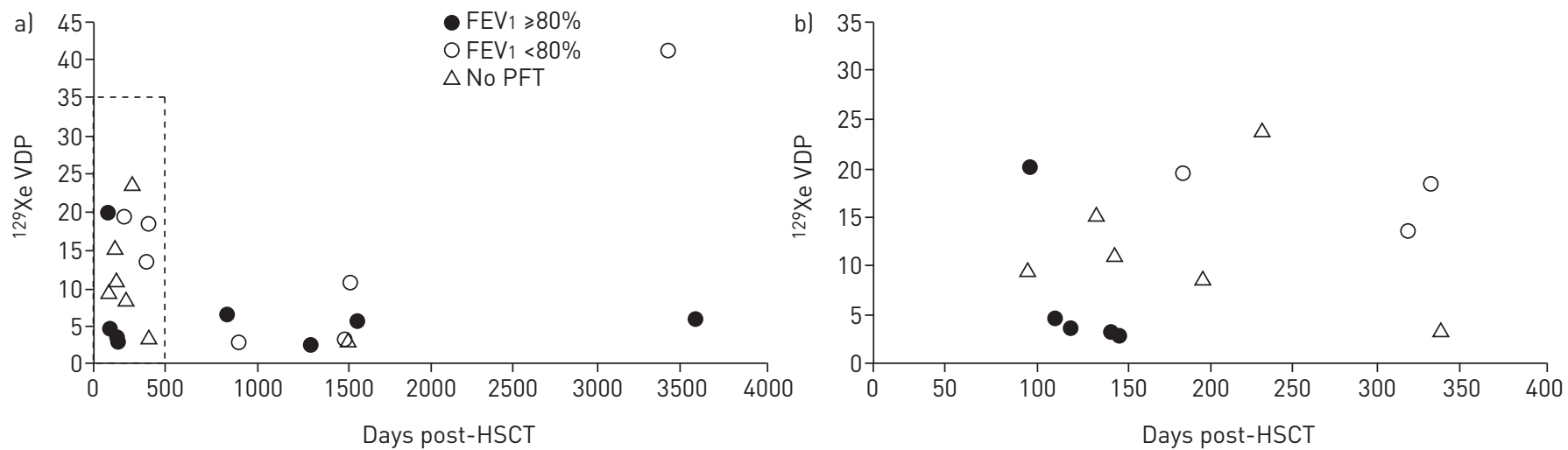

FIGURE 3 a) Xenon-129 $\left({ }^{129} \mathrm{Xe}\right)$ ventilation defect percentage (VDP) plotted against the number of days post-haematopoietic stem cell transplantation (HSCT); b) subjects imaged within the first year (dashed box in al) FEV1: forced expiratory volume in $1 \mathrm{~s}$; PFT: pulmonary function test.

potential of ${ }^{129} \mathrm{Xe}$ MRI continues to be demonstrated across the spectrum of pulmonary disease and patient populations, availability of ${ }^{129} \mathrm{Xe}$ MRI will probably improve. Alongside other known risk factors such as chronic GVHD [46, 47], ventilation deficits on ${ }^{129}$ Xe MRI may inform an algorithm for routine pulmonary screening of HSCT recipients, and as a non-ionising imaging modality, ${ }^{129} \mathrm{Xe}$ MRI is may be used for serial evaluation in this radiation-sensitive paediatric population. In addition to the need for multisite trials of ${ }^{129} \mathrm{Xe}$ MRI with larger study cohorts, future longitudinal studies demonstrating the sensitivity and robustness of ${ }^{129} \mathrm{Xe}$ MRI to early treatment response for individual HSCT patients are in development.

In conclusion, lung ventilation abnormalities following HSCT were quantified using hyperpolarised ${ }^{129} \mathrm{Xe}$ MRI, providing a means of spatially mapping regional lung function without ionising-radiation exposure. While ${ }^{129} \mathrm{Xe}$ VDP was correlated with FEV1 \% pred, FEV1/FVC ratio and $\mathrm{FEF} 25-75 \%$ from spirometry, there was wide variation in ventilation patterns between subjects with similar spirometric parameters, supporting ${ }^{129} \mathrm{Xe}$ as a regional biomarker for individualised assessment of lung abnormalities. There is strong translational potential for ${ }^{129} \mathrm{Xe}$ MRI to personalise treatment approaches for individual HCST patents with pulmonary complications, especially in underevaluated populations such as children who are unable to perform reliable spirometry. ${ }^{129} \mathrm{Xe}$ ventilation MRI may identify asymptomatic patients who should undergo more frequent screening, pre-emptive anti-inflammatory treatment, or be considered for intervention such as bronchoscopy, where the spatial resolution of ${ }^{129} \mathrm{Xe}$ MRI can be leveraged to guide the procedure. The sensitivity of ${ }^{129} \mathrm{Xe}$ to ventilation deficits in asymptomatic HSCT patients with intact spirometry is critical for early intervention to prevent or stabilise disease progression, conserve pulmonary capacity, and ultimately improve outcomes.

Acknowledgements: The authors would like to acknowledge the following Cincinnati Children's Hospital Medical Center (Cincinnati, OH, USA) personnel for their contributions to this study: Erin Watters, Jaylynn Hill and Leann Mount for patient recruitment, coordination and consent; Andrew Bryan and Joseph Plummer for hyperpolarised ${ }^{129} \mathrm{Xe}$ gas preparation; Sharon Kadon and Michelle England for monitoring during the ${ }^{129}$ Xe MRI procedure; and Lacey Haas, Matt Lanier, Brynne Williams, Kaley Bridgewater and Marty Jones for operating the MRI scanner.

This manuscript is dedicated to our late colleague Javier El-Bietar, who touched the lives of many patients.

Author contributions: All authors contributed to the design of the research project, performed the research, and performed the data analysis and interpretation of the results. Javier El-Bietar died in December 2017. All other authors contributed to drafting and approval of the manuscript.

Conflict of interest: L.L. Walkup has nothing to disclose. K. Myers reports personal fees from Novartis and Bellicum, outside the submitted work. A. Nelson has nothing to disclose. M.M. Willmering has nothing to disclose. M. Grimley has nothing to disclose. S.M. Davies has nothing to disclose. C. Towe has nothing to disclose. J.C. Woods reports grants from and consultancy for Vertex Pharmaceuticals, and grants from Grifols, Inc., outside the submitted work.

Support statement: This work was supported by NIH K99HL138255 and T32HL007752. Funding information for this article has been deposited with the Crossref Funder Registry.

\section{References}

1 Sakaida E, Nakaseko C, Harima A, et al. Late-onset noninfectious pulmonary complications after allogeneic stem cell transplantation are significantly associated with chronic graft-versus-host disease and with the graft-versusleukemia effect. Blood 2003; 102: 4236-4242.

2 Kotloff RM, Ahya VN, Crawford SW. Pulmonary complications of solid organ and hematopoietic stem cell transplantation. Am J Respir Crit Care Med 2004; 170: 22-48. 
3 Fidler MM, Reulen RC, Bright CJ, et al. Respiratory mortality of childhood, adolescent and young adult cancer survivors. Thorax 2018; 73: 959-968.

4 Williams KM. How I treat bronchiolitis obliterans syndrome after hematopoietic stem cell transplantation. Blood 2017; 129: 448-455.

5 Cheng GS, Storer B, Chien JW, et al. Lung function trajectory in bronchiolitis obliterans syndrome after allogeneic hematopoietic cell transplant. Ann Am Thorac Soc 2016; 13: 1932-1939.

6 Dharmagunawardena RT, Pearce RM, Lee J, et al. S135 A retrospective multi-centre study of the effects of allogeneic haematopoietic stem cell transplantation on pulmonary function. Thorax 2011; 66: Suppl. 4, A62.

7 Uhlving HH, Bang CL, Christensen IJ, et al. Lung function after allogeneic hematopoietic stem cell transplantation in children: a longitudinal study in a population-based cohort. Biol Blood Marrow Transplant 2013; 19: $1348-1354$.

8 Cooke KR. A "window of opportunity" for patients with late-onset pulmonary dysfunction after allogeneic hematopoietic cell transplantation. Biol Blood Marrow Transplant 2014; 20: 291-292.

9 Palmer J, Williams K, Inamoto Y, et al. Pulmonary symptoms measured by the National Institutes of Health lung score predict overall survival, nonrelapse mortality, and patient-reported outcomes in chronic graft-versus-host disease. Biol Blood Marrow Transplant 2014; 20: 337-344.

10 Gustafsson PM, De Jong PA, Tiddens HA, et al. Multiple-breath inert gas washout and spirometry versus structural lung disease in cystic fibrosis. Thorax 2008; 63: 129-134.

11 Horsley AR, Gustafsson PM, Macleod KA, et al. Lung clearance index is a sensitive, repeatable and practical measure of airways disease in adults with cystic fibrosis. Thorax 2008; 63: 135-140.

12 Carpenter PA, Kitko CL, Elad S, et al. National Institutes of Health consensus development project on criteria for clinical trials in chronic graft-versus-host disease: V. The 2014 Ancillary Therapy and Supportive Care working group report. Biol Blood Marrow Transplant 2015; 21: 1167-1187.

13 Majhail NS, Rizzo JD, Lee SJ, et al. Recommended screening and preventive practices for long-term survivors after hematopoietic cell transplantation. Bone Marrow Transplant 2012; 47: 337-341.

14 Prais D, Sinik MM, Stein J, et al. Effectiveness of long-term routine pulmonary function surveillance following pediatric hematopoietic stem cell transplantation. Pediatr Pulmonol 2014; 49: 1124-1132.

15 Loeb JS, Blower WC, Feldstein JF, et al. Acceptability and repeatability of spirometry in children using updated ATS/ERS criteria. Pediatr Pulmonol 2008; 43: 1020-1024.

16 Gazourian L, Coronata AMF, Rogers AJ, et al. Airway dilation in bronchiolitis obliterans after allogeneic hematopoietic stem cell transplantation. Respir Med 2013; 107: 276-283.

17 Togni Filho PH, Casagrande JLM, Lederman HM. Utility of the inspiratory phase in high-resolution computed tomography evaluations of pediatric patients with bronchiolitis obliterans after allogeneic bone marrow transplant: reducing patient radiation exposure. Radiol Bras 2017; 50: 90-96.

18 Thomen RP, Walkup LL, Roach DJ, et al. Hyperpolarized ${ }^{129} \mathrm{Xe}$ for investigation of mild cystic fibrosis lung disease in pediatrics. J Cyst Fibros 2017; 16: 275-282.

19 Kanhere N, Couch MJ, Kowalik K, et al. Correlation of lung clearance index with hyperpolarized ${ }^{129}$ Xe magnetic resonance imaging in pediatric subjects with cystic fibrosis. Am J Respir Crit Care Med 2017; 196: 1073-1075.

20 Thomen RP, Sheshadri A, Quirk JD, et al. Regional ventilation changes in severe asthma after bronchial thermoplasty with ${ }^{3} \mathrm{He}$ MR imaging and CT. Radiology 2015; 274: 250-259.

21 Qing K, Mugler JP 3rd, Altes TA, et al. Assessment of lung function in asthma and COPD using hyperpolarized ${ }^{129} \mathrm{Xe}$ chemical shift saturation recovery spectroscopy and dissolved-phase MRI. NMR Biomed 2014; 27: $1490-1501$

22 de Lange EE, Altes TA, Patrie JT, et al. Evaluation of asthma with hyperpolarized helium-3 MRI: correlation with clinical severity and spirometry. Chest 2006; 130: 1055-1062.

23 Wang JM, Robertson SH, Wang Z, et al. Using hyperpolarized ${ }^{129}$ Xe MRI to quantify regional gas transfer in idiopathic pulmonary fibrosis. Thorax 2018; 73: 21-28.

24 Kirby M, Svenningsen S, Kanhere N, et al. Pulmonary ventilation visualized using hyperpolarized helium-3 and xenon-129 magnetic resonance imaging: differences in COPD and relationship to emphysema. J Appl Physiol 2013; 114: 707-715.

25 Driehuys B, Martinez-Jimenez S, Cleveland ZI, et al. Chronic obstructive pulmonary disease: safety and tolerability of hyperpolarized Xe-129 MR imaging in healthy volunteers and patients. Radiology 2012; 262: 279-289.

26 Stewart NJ, Chan HF, Hughes PJC, et al. Comparison of ${ }^{3} \mathrm{He}$ and ${ }^{129} \mathrm{Xe}$ MRI for evaluation of lung microstructure and ventilation at 1.5T. J Magn Reson Imaging 2018; 48: 632-642.

27 O'Sullivan B, Couch M, Roche JP, et al. Assessment of repeatability of hyperpolarized gas MR ventilation functional imaging in cystic fibrosis. Acad Radiol 2014; 21: 1524-1529.

28 Safavi S, Arthofer C, Thorpe J, et al. Reproducibility of hyperpolarised xenon-129 MRI ( $\left.{ }^{129} \mathrm{Xe}-\mathrm{MRI}\right)$ of lungs in healthy volunteers. Eur Respir J 2017; 50: Suppl. 61, PA3743.

29 McAdams HP, Palmer SM, Donnelly LF, et al. Hyperpolarized ${ }^{3} \mathrm{He}$-enhanced MR imaging of lung transplant recipients: preliminary results. AJR Am J Roentgenol 1999; 173: 955-959.

30 Gast KK, Biedermann A, Herweling A, et al. Oxygen-sensitive ${ }^{3} \mathrm{He}-\mathrm{MRI}$ in bronchiolitis obliterans after lung transplantation. Eur Radiol 2008; 18: 530-537.

31 Walkup LL, Myers KC, El-Bietar J, et al. Regional lung ventilation deficits in pediatric hematopoietic stem cell transplant patients revealed via hyperpolarized xenon-129 magnetic resonance imaging. Biol Blood Marrow Transplant 2018; 24: S66.

32 Walkup LL, Myers K, El-Bietar J, et al. Hyperpolarized 129Xe MRI reveals early regional ventilation deficits in pediatric hematopoietic stem cell transplant recipients. Am J Respir Crit Care Med 2018; 197: A2348.

33 Stocks J, Quanjer PH. Reference values for residual volume, functional residual capacity and total lung capacity. ATS Workshop on Lung Volume Measurements. Official Statement of The European Respiratory Society. Eur Respir J 1995; 8: 492-506.

34 Walkup LL, Thomen RP, Akinyi T, et al. Feasibility, tolerability, and safety of pediatric hyperpolarized ${ }^{129}$ Xe magnetic resonance imaging in healthy volunteers and children with cystic fibrosis. Pediatr Radiol 2016; 46: 1651-1662.

35 Kirby M, Heydarian M, Svenningsen S, et al. Hyperpolarized $3 \mathrm{He}$ magnetic resonance functional imaging semiautomated segmentation. Acad Radiol 2012; 19: 141-152. 
Santyr G, Kanhere N, Morgado F, et al. Hyperpolarized gas magnetic resonance imaging of pediatric cystic fibrosis lung disease. Acad Radiol 2019; 26: 344-354.

37 He M, Kaushik SS, Robertson SH, et al. Extending semiautomatic ventilation defect analysis for hyperpolarized ${ }^{129}$ Xe ventilation MRI. Acad Radiol 2014; 21: 1530-1541.

38 Kirby M, Svenningsen S, Owrangi A, et al. Hyperpolarized 3He and 129Xe MR imaging in healthy volunteers and patients with chronic obstructive pulmonary disease. Radiology 2012; 265: 600-610.

39 Virgincar RS, Cleveland ZI, Kaushik SS, et al. Quantitative analysis of hyperpolarized ${ }^{129}$ Xe ventilation imaging in healthy volunteers and subjects with chronic obstructive pulmonary disease. NMR Biomed 2013; 26: 424-435.

40 Thomen RP, Walkup LL, Roach DJ, et al. Hyperpolarized ${ }^{129} \mathrm{Xe}$ for investigation of mild cystic fibrosis lung disease in pediatric patients. J Cyst Fibros 2017; 16: 275-282.

41 Shukla Y, Wheatley A, Kirby M, et al. Hyperpolarized ${ }^{129}$ Xe magnetic resonance imaging: tolerability in healthy volunteers and subjects with pulmonary disease. Acad Radiol 2012; 19: 941-951.

42 Yamashiro T, Iida G, Kamiya $\mathrm{H}$, et al. Scintigraphy and computed tomography findings for the diagnosis of bronchiolitis obliterans following peripheral blood stem cell transplantation. Hell J Nucl Med 2012; 15: 52-55.

43 Johns DP, Walters JAE, Walters EH. Diagnosis and early detection of COPD using spirometry. J Thorac Dis 2014; 6: 1557-1569.

44 Bhatt SP, Soler X, Wang X, et al. Association between functional small airway disease and FEV 1 decline in chronic obstructive pulmonary disease. Am J Respir Crit Care Med 2016; 194: 178-184.

45 Srinivasan A, Srinivasan S, Sunthankar S, et al. Pre-hematopoietic stem cell transplant lung function and pulmonary complications in children. Ann Am Thorac Soc 2014; 11: 1576-1585.

46 Grkovic L, Pulanic D, Steinberg SM, et al. Chronic graft-versus-host disease (cGVHD) patients with bronchiolitis obliterans syndrome (BOS) have worse clinical manifestations and severity of cGVHD with more impairment in self-assessed physical and mental health than cGVHD patients without BOS. Blood 2012; 120: 1939-1939.

47 Neji HB, Dromer C, Tabrizi R, et al. Bronchiolitis obliterans after allogeneic stem cell transplantation: a single center retrospective study of risk factors. Blood 2010; 116: 4507-4507. 